\title{
RATE Distortion STUdy For TIME- VARYING AUTOREGRESSIVE GAUSSIAN PROCESS
}

\author{
Jia-Chyi Wu \\ Department of Communications, Control and Navigation Engineering, \\ National Taiwan Ocean University, Keelung, Taiwan, ROC
}

\begin{abstract}
Formulation of the Rate-distortion association is an information-theoretic study in the field of signal encoding systems. Since a more general approach to model the nonstationarity exhibited by real-world signals is to use appropriately fitted time varying autoregressive (TVAR) models, we have investigated the rate-distortion function $R(D)$ for the class of time varying nonstationary signals. In this study, we present formulations of the rate-distortion function for the Gaussian TVAR processes. The $R(D)$ function can serve as an information-theoretic bound on the performance achievable by source encoding techniques when the processing signal is represented exclusively by a Gaussian TVAR model.
\end{abstract}

\section{KEYWORDS}

Rate Distortion, Nonstationary, Time Varying Autoregressive (TVAR) Process

\section{INTRODUCTION}

Rate-distortion analysis was first suggested in Shannon's original development of information theory [1] and it was exclusively developed for memoryless and Markov sources in conjunction with the squared-error distortion measure [2]. It was later generalized to stationary ergodic processes with discrete alphabets and to Gaussian processes by Gallager [3], as well as to stationary and abstract alphabets by Berger [4]. Many contributions have been made to ratedistortion theory for varied stationary sources [5]-[6], [17]-[23]. The theory is comprehensively discussed in books by Berger [4] and Gallager [3]. Extensive bibliographies for this subject appear in [7]-[9]. Recently, Jesús Gutiérrez-Gutiérrez, et. al. present an integral formula for the rate-distortion function of asymptotically wide sense stationary (AWSS) Gaussian vector process as well as the $R(D)$ function of moving average (MA) Gaussian vector processes and of autoregressive MA (ARMA) AWSS Gaussian vector processes [24]-[25]. Due to the nonstationary characteristics of real-world signal sources, recent research interest has focused on extending the $R(D)$ function for the nonstationary process. Berger [10] proved a source coding theorem with respect to a squared-error distortion measure for the Wiener process, a special case of the nonstationary Gaussian AR process. Gray [11] showed that the source coding theorem generally holds for the Gaussian AR process even if the process is neither stationary nor asymptotically stationary. Hashimoto and Arimoto [12] then developed a general form for the $R(D)$ function for the class of a nonstationary Gaussian AR process wherein the variance of the process grows exponentially. However, the nonstationary Gaussian AR process indicated in these papers [10]-[12] becomes unstable asymptotically, since the variance of the AR process grows either exponentially or algebraically. Since the nonstationary characteristics exhibited by realworld signals can be modelled using appropriately fitted time varying autoregressive (TVAR)

Natarajan Meghanathan et al. (Eds) : CSITEC, CMCA, SP, NECOM, ADCO - 2019 
forms [13]-[14], we have investigated the $R(D)$ formulation for the class of time varying nonstationary signals in this study.

In this paper we present the $R(D)$ function study for Gaussian TVAR processes. First of all, we briefly review the $R(D)$ function for time-discrete sources that are input to a general communication system. We then present the methodology to form the $R(D)$ function for Gaussian TVAR processes. The $R(D)$ function is an ultimate bound in assessing both the absolute and relative performance achievable by time varying signal source coding system.

\section{Preliminary Review for $R(D)$ Function}

The rate-distortion function, $R(D)$, is mathematically defined in terms of the average mutual information between the source outputs and the reproduced source outputs at the destination. Consider a time-discrete, continuous-amplitude, stationary source $\left\{x_{t}, t=\ldots,-1,0,1, \ldots\right\}$ having a joint probability density $P(\mathbf{x})=P\left(x_{1}, x_{2}, \ldots, x_{N}\right)$ governing the generation of $N$ successive source letters. Also, specify a single-letter fidelity criterion $F$, which is the distortion between a source word $x$ and a reproducing word $y$ present at the destination, to be defined as the arithmetic average of the distortions between the corresponding letters of $\mathbf{x}$ and $\mathbf{y}$ as assigned by a fixed single-letter nonnegative distortion measure, $\rho\left(x_{t}, y_{t}\right)$. Consider all possible conditional probability densities $Q(\mathbf{y} \mid \mathbf{x})$ associated with reproducing alphabet $\mathbf{y}=\left\{y_{1}, y_{2}, \ldots, y_{N}\right\}$ and the given source alphabet $\mathbf{x}=\left\{x_{1}, x_{2}, \ldots, x_{N}\right\}$.

The $R(D)$ function for the particular source $\left\{x_{t}\right\}$ with respect to $F_{\rho}$ is defined as

$$
\begin{aligned}
& R(D)=\lim _{N \rightarrow \infty} R_{N}(D), \\
& R_{N}(D)=\frac{1}{N} \inf _{Q \in Q_{D}} I(Q),
\end{aligned}
$$

where $I(Q)$ is the average mutual information, defined as a function of the conditional probability density function. The limit in Eq. (1) always exists for a stationary source [4] so that $R(D)$ is welldefined. In the particular case of a one-dimensional time-discrete stationary Gaussian source with power spectral density (PSD)

$$
S(\omega)=\sum_{k=-\infty}^{\infty} c_{k} e^{-j k \omega},
$$

where $c_{k}$ is the autocorrelation function, and using the asymptotic form of $R(D)$ in Eq. (1), it can be shown that the mean-squared error (MSE) rate-distortion function has the parametric representation [4],

$$
\begin{gathered}
D_{\theta}=\frac{1}{2 \pi} \int_{-\pi}^{\pi} \min [\theta, S(\omega)] d \omega, \\
R\left(D_{\theta}\right)=\frac{1}{2 \pi} \int_{-\pi}^{\pi} \max \left[0, \frac{1}{2} \log \left(\frac{S(\omega)}{\theta}\right)\right] d \omega,
\end{gathered}
$$

with the non-zero portion of the $R(D)$ curve being generated by the parameter $\theta$ in the interval $0 \leq \theta \leq$ ess sup $S(\omega)$, where the arithmetic average squared error is defined as $\left(y_{t}-x_{t}\right)^{2}$. The $R(D)$ 
function for a Gaussian source with memory is completely specified in terms of the source properties, namely the source power spectral density.

\section{3. $R(D)$ FUNCTION FOR GAUSSIAN TVAR Process}

The Gaussian time-varying AR process is of primary interest because of its wide applicability to the modeling of real-world sources, e.g., speech signals and rasterized image signals. In this section, we consider the one-dimensional Gaussian TVAR process. Let $\left\{x_{t}\right\}$ be a Gaussian TVAR process satisfying the difference equation

$$
x_{t}=-\sum_{m=1}^{M} a_{m}\left(\frac{t}{N}\right) x_{t-m}+z_{t}, \text { for } t=1,2, \ldots, N,
$$

where $x_{t}=0$ for $t=0,-1,-2, \ldots$, and $z_{t}$ are independent and identically distributed (i.i.d.) zeromean Gaussian random variables possessing variance $\sigma_{z}^{2}$. The terms $a_{m}\left(\frac{t}{N}\right)$ are the AR parameters varying with time $t=1,2, \ldots, N$. The time index $\left(\frac{t}{N}\right)$ of the time-varying AR parameters $a_{m}\left(\frac{t}{N}\right)$ is defined such that $0<\left(\frac{t}{N}\right) \leq 1$.

Upon iterating Eq. (6) to obtain $\mathbf{x}=\left\{x_{1}, x_{2}, \ldots, x_{N}\right\}$, it is observed that $\mathbf{x}$ is specified uniquely by $\mathbf{z}$ $=\left\{z_{1}, z_{2}, \ldots, z_{N}\right\}$ and the known initial state $\mathbf{x}_{0}=\left\{x_{0}, x_{-1}, \ldots, x_{-m+1}\right\}=(0,0, \ldots, 0)$. Therefore, $z_{t}$ can be specified uniquely by $\mathbf{x}$ and $\mathbf{x}_{0}$ via the relation

$$
z_{t}=\sum_{m=0}^{M} a_{m}\left(\frac{t}{N}\right) x_{t-m}, \text { for } t=1,2, \ldots, N
$$

with $a_{0}()=$.1 . Equation (7) can be represented in matrix form, $\mathbf{z}=\mathbf{A x}$, for $t=1,2, \ldots, N$, where $\mathbf{A}$ is the $N \times N$ lower triangular matrix,

$$
\mathbf{A}=\left[\begin{array}{ccccccc}
a_{0}\left(\frac{1}{N}\right) & 0 & 0 & \cdots & \cdots & \cdots & 0 \\
a_{1}\left(\frac{2}{N}\right) & a_{0}\left(\frac{2}{N}\right) & 0 & \cdots & \cdots & \cdots & 0 \\
\vdots & \vdots & \ddots & \ddots & \vdots & \vdots & \vdots \\
a_{M}\left(\frac{M+1}{N}\right) & a_{M-1}\left(\frac{M+1}{N}\right) & \cdots & a_{0}\left(\frac{M+1}{N}\right) & 0 & \cdots & 0 \\
0 & a_{M}\left(\frac{M+2}{N}\right) & \cdots & \cdots & a_{0}\left(\frac{M+2}{N}\right) & \ldots & 0 \\
\vdots & \vdots & \vdots & \vdots & \vdots & \vdots & \vdots \\
0 & \vdots & 0 & a_{M}\left(\frac{N-1}{N}\right) & \cdots & a_{0}\left(\frac{N-1}{N}\right) & 0 \\
0 & \cdots & \cdots & 0 & a_{M}(1) & \cdots & a_{0}(1)
\end{array}\right] .
$$

For the Gaussian AR process $\left\{x_{t}\right\}$, let $R_{N}(D)$ be the per letter rate-distortion function of the $n$ dimensional vector $\left(x_{1}, x_{2}, \ldots, x_{N}\right)$. The rate-distortion function of the process $\left\{x_{t}\right\}$ is defined as

$$
R(D)=\lim _{N \rightarrow \infty} R_{N}(D)
$$

and from Berger [4] (6.3.34) and (6.3.35), $R_{N}(D)$ has the following parametric form when $x_{t}$ is an $N$-dimensional zero-mean Gaussian distributed random vector 


$$
\begin{aligned}
& D_{\theta}=\frac{1}{N} \sum_{m=0}^{N} \min \left(\theta, \frac{1}{\alpha_{m}}\right) \\
& R_{N}\left(D_{\theta}\right)=\frac{1}{N} \sum_{m=0}^{N} \max \left(0, \frac{1}{2} \log \frac{1}{\theta \alpha_{m}}\right)
\end{aligned}
$$

where, $\alpha_{m}$ are the eigenvalues of the inverse autocorrelation matrix $\boldsymbol{\Phi}_{N}^{-1}$ of the random variables $x_{1}, x_{2}, \ldots, x_{N}$, and $\theta$ is a parameter taking on values in the interval $0 \leq \theta<\alpha_{\max }$. From the relationship $\mathbf{z}=\mathbf{A} \mathbf{x}$, we then have

$$
\boldsymbol{\Phi}_{N}=E\left[\mathbf{X} \mathbf{X}^{T}\right]=\mathbf{A}^{-1} E\left[\mathbf{z z}^{T}\right]\left(\mathbf{A}^{T}\right)^{-1}=\sigma_{z}^{2}\left(\mathbf{A}^{T} \mathbf{A}\right)^{-1},
$$

and $\mathbf{A}^{-1}$ exists because

$$
\operatorname{det} \mathbf{A}=\prod_{t=1}^{N} a_{0}\left(\frac{t}{N}\right)=1 \neq 0
$$

Hence, the inverse autocorrelation matrix is found to be

$$
\boldsymbol{\Phi}_{N}^{-1}=\left(\frac{1}{\sigma_{z}^{2}}\right) \mathbf{A}^{T} \mathbf{A}
$$

The entries in the inverse matrix $\boldsymbol{\Phi}_{N}^{-1}$ are given as $\phi_{N}^{-1}(\mu, v)$, and

$$
\phi_{N}^{-1}(\mu, v)=\frac{1}{\sigma_{z}^{2}} \sum_{m=0}^{N-\max (\mu, v)} a_{m}\left(\frac{m+\max (\mu, v)}{N}\right) a_{m+|\mu-v|}\left(\frac{m+\max (\mu, v)}{N}\right)
$$

Since $a_{m}()=$.0 for $m>M$, it follows that all entries more than $M$ diagonals away from the main diagonal of $\boldsymbol{\Phi}_{N}^{-1}$ are zero. Also, if either $\mu \leq N-M$ or $v \leq N-M$, then $\phi_{N}^{-1}(\mu, v)=\phi_{N}^{-1}(|\mu-v|)$, and

$$
\phi_{N}^{-1}(|\mu-v|)=\frac{1}{\sigma_{z}^{2}} \sum_{m=0}^{M} a_{m}\left(\frac{m+\max (\mu, \nu)}{N}\right) a_{m+|\mu-v|}\left(\frac{m+\max (\mu, \nu)}{N}\right)
$$

Hence $\boldsymbol{\Phi}_{N}^{-1}$ is a Hermitian matrix. Due to the Hermitian structure of $\boldsymbol{\Phi}_{N}^{-1}$, it is possible to consider the limiting case of infinite $N$ in Eq. (10). By invoking a theorem defined by Grenander and Szegö [15] regarding the asymptotic distribution of the eigenvalues of certain Hermitian structures, we first consider the class of real-valued functions $f(r, \omega), 0 \leq r \leq 1$, periodic in $\omega$ with period $2 \pi$, satisfying the following condition,

\section{Condition:}

The coefficients $\psi_{n}(r)$ of the Fourier series

$$
f(r, \omega)=\sum_{n=-\infty}^{\infty} \psi_{n}(r) e^{-j n \omega}
$$

are continuous and there exists a constant $K$ such that

$$
\sum_{n=-\infty}^{\infty} \max \left|\psi_{n}(r)\right| \leq K,
$$

where the maximum values are taken in the interval $0 \leq r \leq 1$. 
The Fourier series of $f(r, \omega)$ is absolutely convergent and the function $f(r, \omega)$ of the two variables $r$ and $\omega$ is continuous. We then have the following theorem,

Theorem I. Asymptotic distribution theorem of the eigenvalues of the specified Hermitian form (ref. Grenander and Szegö [15], Theorem 6.5)

Let the function $f(r, \omega)$, where $r=\frac{\max (\mu, v)}{N}$, satisfy the above condition. We denote the eigenvalues of the Hermitian form

$$
\sum_{\mu, v} \psi_{v-\mu}\left(\frac{\max (\mu, v)}{N}\right) u_{\mu} u_{v}, \mu, v=0,1,2, \ldots, N
$$

by $\lambda_{v}^{(N)}$, we have then $\left|\lambda_{v}^{(N)}\right| \leq K$. Moreover, if $F(\lambda)$ is any continuous function defined for $-K \leq$ $\lambda \leq K$, we have

$$
\lim _{N \rightarrow \infty} \frac{1}{N} \sum_{v=1}^{N} F\left(\lambda_{v}^{(N)}\right)=\frac{1}{2 \pi} \int_{-\pi}^{\pi} \int_{0}^{1} F[f(r, \omega)] d r d \omega .
$$

In the special case when $f(r, \omega)$ is independent of $r$, this is an assertion on Toeplitz forms. The limit relation Eq. (17) is equivalent to the following special case [15],

$$
\lim _{N \rightarrow \infty} \frac{1}{N} \sum_{v=1}^{N}\left(\lambda_{v}^{(N)}\right)^{k}=\frac{1}{2 \pi} \int_{-\pi}^{\pi} \int_{0}^{1}[f(r, \omega)]^{k} d r d \omega, \quad k=0,1,2, \ldots
$$

Theorem I can be attested directly from the procedures described both in Grenander and Szegö [15] Theorem 1.18(b) and Theorem 6.5.

Since we have defined $r=\frac{\max (\mu, v)}{N}, \mu, v=1,2, \ldots, N, \phi_{N}^{-1}(\mu, v)$ is rewritten as

$$
\phi_{N}^{-1}(|\mu-v|)=\phi_{\mu-v \mid}^{-1}(r)=\frac{1}{\sigma_{z}^{2}} \sum_{m=0}^{M} a_{m}\left(\frac{m}{N}+r\right) a_{m+|\mu-v|}\left(\frac{m}{N}+r\right) .
$$

Now, let $\mathbf{G}_{N}=\boldsymbol{\Phi}_{N}^{-1}$ and $\left\{\mathbf{G}_{N}\right\}$ be sequences of Hermitian matrices with eigenvalues $\left\{\alpha_{m}^{(N)}\right\}$. The entries of the symmetric Hermitian matrix $\mathbf{G}_{N}$ are

$$
g_{|\mu-v|}(r)=g_{k}(r)=\frac{1}{\sigma_{z}^{2}} \sum_{m=0}^{M} a_{m}\left(\frac{m}{N}+r\right) a_{m+|\mu-v|}\left(\frac{m}{N}+r\right)
$$

on the $k^{\text {th }}$ diagonal. In most real-world signal processing cases, the autoregressive order $M$ is much less than $N$, therefore, we have that $m / N \rightarrow 0$ when $N \rightarrow \infty$. Equation (21) is simplified as

$$
g_{k}(r)=\frac{1}{\sigma_{z}^{2}} \sum_{m=0}^{M} a_{m}(r) a_{m+k}(r) .
$$

Since the $\mathbf{G}_{N}$ possess the Hermitian form specified by Eq. (17), Theorem I implies that their eigenvalues $\left\{\alpha_{m}^{(N)}\right\}$ are distributed asymptotically according to 


$$
g(r, \omega)=\sum_{k=-\infty}^{\infty} g_{k}(r) e^{-j k \omega}=\frac{1}{\sigma_{z}^{2}}\left|\sum_{m=0}^{M} a_{m}(r) e^{-j m \omega}\right|^{2}
$$

with $\omega$ uniform on $[-\pi, \pi]$, and that

$$
\lim _{N \rightarrow \infty}\left|\mathbf{G}_{N}\right|=\sqrt{\frac{1}{2 \pi} \int_{-\pi}^{\pi} \int_{0}^{1} g^{2}(r, \omega) d r d \omega}<\infty
$$

where $\left|\mathbf{G}_{N}\right|$ represents the weak norm of $\mathbf{G}_{N}$,

$$
\left|\mathbf{G}_{N}\right|=\sqrt{\frac{1}{N} \sum_{\mu, v=1}^{N}\left|G_{N}(\mu, v)\right|^{2}}=\sqrt{\frac{1}{N} \sum_{m=1}^{N}\left(\alpha_{m}^{(N)}\right)^{2}} .
$$

The integral in Eq. (24) is finite due to the fact that

$$
0 \leq g(r, \omega) \leq \frac{1}{\sigma_{z}^{2}}\left(\sum_{m=0}^{M}\left|a_{m}(r)\right|\right)^{2}<\infty .
$$

Now, by applying Theorem I, we have that

$$
\lim _{N \rightarrow \infty} \frac{1}{N} \sum_{m=1}^{N} F\left(\alpha_{m}^{(N)}\right)=\frac{1}{2 \pi} \int_{-\pi}^{\pi} \int_{0}^{1} F[g(r, \omega)] d r d \omega
$$

for any continuous function $F$ on $[\delta, \mu]$, where $\delta$ and $\mu$ are the essential infimum and supremum of $g(r, \omega)$, respectively. Applying Eq. (27) so as to pass to the limit in Eq. (10), we obtain the following theorem as a consequence.

\section{Theorem II. Rate-distortion function for time-varying autoregressive Gaussian process}

Let $x_{t}$ be an $M^{\text {th }}$-order Gaussian TVAR source generated by an i.i.d. $N\left(0, \sigma_{z}^{2}\right)$ sequence $z_{t}$ and the TVAR coefficients $a_{m}(r), m=1,2, \ldots, M$, where $r=\frac{t}{N}$. Then the mean-squared error (MSE) ratedistortion function of $x_{t}$ is given parametrically by

$$
\begin{aligned}
& D_{\theta}=\frac{1}{2 \pi} \int_{-\pi}^{\pi} \int_{0}^{1} \min \left[\theta, \frac{1}{g(r, \omega)}\right] d r d \omega, \\
& R\left(D_{\theta}\right)=\frac{1}{2 \pi} \int_{-\pi}^{\pi} \int_{0}^{1} \max \left[0, \frac{1}{2} \log \frac{1}{\theta g(r, \omega)}\right] d r d \omega,
\end{aligned}
$$

where

$$
g(r, \omega)=\frac{1}{\sigma_{z}^{2}}\left|1+\sum_{m=1}^{M} a_{m}(r) e^{-j m \omega}\right|^{2}
$$




\section{SUMmary AND CONCluSIONS}

The rate-distortion function $R(D)$ for time-varying autoregressive (TVAR) nonstationary signals, based upon the theorem from Grenander and Szegö [15] regarding the asymptotic distribution of the eigenvalues of certain Hermitian forms, is investigated and formulated in this study. The $R(D)$ function is served as an information-theoretic bound on the performance achievable by source encoding techniques when the processing signal is represented exclusively by a Gaussian TVAR model. The rate-distortion function can be used as an ultimate performance bound in assessing both the absolute and relative performance achievable by any specific time-varying signal processing system.

\section{ACKNOWLEDGEMENTS}

This work has been supported partially by the Ministry of Science and Technology in Taiwan, the Republic of China under grant number MOST 107-2119-M-019-004.

\section{REFERENCES}

[1] C. E. Shannon, (1948) "A mathematical theory of communication," Bell Syst. Tech. Journal, vol. 27, pp. 379-423 and pp. 623-656.

[2] C. E. Shannon, (1959) "Coding theorems for a discrete source with a fidelity criterion," IRE Natl. Cov. Rec., Part 4, pp. 142-163.

[3] R. G. Gallagher, (1968) Information Theory and Reliable Communications. New York: Wiley \& Sons, ch. 9.

[4] T. Berger, (1971) Rate Distortion Theory: A Mathematical Basis for Data Compression. Englewood Cliffs, NJ: Prentice Hall.

[5] R. E. Blahut, (1972) "Computation of channel capacity and rate-distortion functions," IEEE Trans. on Inform. Theory, vol. IT-18, pp. 460-473.

[6] L. D. Davisson, (1972) "Rate-distortion theory and application," Proceedings of IEEE, vol. 60, pp. 800-808.

[7] H. C. Andrews, (1971) "Bibliography on rate distortion theory," IEEE Trans. on Inform. Theory, vol. IT-17, pp. 198-199.

[8] L. C. Wilkins and P. A. Wintz, (1971) "Bibliography on data compression, picture properties, and picture coding," IEEE Trans. on Inform. Theory, vol. IT-17, pp. 180-197.

[9] J. C. Kieffer, (1993) "A survey of the theory of source coding with a fildelity criterion," IEEE Trans. on Inform. Theory, vol. IT-39, pp. 1473-1490.

[10] T. Berger, (1970) "Information rates of Wiener processes," IEEE Trans. on Inform. Theory, vol. IT16, pp. 134-139.

[11] R. M. Gray, (1970) "Information rates of autoregressive processes," IEEE Trans. on Inform. Theory, vol. IT-16, pp. 412-421.

[12] T. Hashimoto and S. Arimoto, (1980) "On the rate-distortion function for the nonstationary Gaussian autoregressive process," IEEE Trans. on Inform. Theory, vol. IT-26, pp. 478-480. 
[13] G. Alengrin, M. Barlaud, and J. Menez, (1986) "Unbiased parameter estimation of nonstationary signals in noise," IEEE Trans. on Acoust., Speech, Signal Processing, vol. 34, pp. 319-1322.

[14] Y. Grenier, (1983) "Time-dependent ARMA modeling of nonstationary signals," IEEE Trans. on Acoust., Speech, Signal Processing, vol. ASSP-31, pp. 899-911.

[15] U. Grenander and G. Szegö, (1958) Toeplitz Forms and Their Applications, CA: Univ. of Cal. Press.

[16] Y. Steinberg and S. Verdú, (1996) "Simulation of random processes and rate-distortion theory," IEEE Trans. on Inform. Theory, vol. IT-42, pp. 63-86.

[17] J. Muramatsu and F. Kanaya, (1994) "Distortion-complexity and rate-distortion function," IEICE Trans. Fundamentals, Vol.E77-A, No.8, pp.1224-1229.

[18] F. Kanaya and J. Muramatsu, (1997) "An almost sure recurrence theorem with distortion for stationary ergodic sources,” IEICE Trans. Fundamentals, E80-A, No.11, pp.2264-2267.

[19] K. Iwata and J. Muramatsu, (2002) “An information-spectrum approach to rate-distortion function with side information,” IEICE Trans. Fundamentals, Vol.E85-A, No.6, pp.1387-1395.

[20] T. M. Cover and M. Chiang, (2002) "Duality between channel capacity and rate distortion with twosided state information,” IEEE Trans. on Inform. Theory, vol. IT-48, pp. 1629-1638.

[21] S. Cheng, V. Stankovic, Z. Xiong, (2005) "Computing the channel capacity and rate-distortion function with two-sided state information," IEEE Trans. on Info. Theory, vol. 51, pp. 4418-4425.

[22] M. Fleming and M. Effros, (2006) "On the rate-distortion with mixed types of side information", IEEE Trans. on Inform. Theory, vol. IT-52, pp. 1698-1705.

[23] J. Binia and H. Israel, (2008) "Rate distortion function with a proportional mean-square error distortion measure," in Proceedings of the International Symposium on Information Theory (ISIT), pp. 862-866, Toronto, Canada.

[24] Gutiérrez-Gutiérrez, J.; Zárraga-Rodríguez, M.; Villar-Rosety, F.M.; Insausti, X., (2018) "RateDistortion Function Upper Bounds for Gaussian Vectors and Their Applications in Coding AR Sources," Entropy, Vol. 20, 399.

[25] Gibson, J., (2017) "Rate Distortion Functions and Rate Distortion Function Lower Bounds for RealWorld Sources” Entropy, Vol. 19, 604.

\section{AUTHORS}

Jia-Chyi Wu (Ph.D., Rutgers, the state University of New Jersey, USA) is an Assistant Professor at Department of Communications, Control and Navigation Engineering, National Taiwan Ocean University, Keelung, Taiwan, ROC. His areas of research include image and video signal coding, digital communications design, and information coding theory.

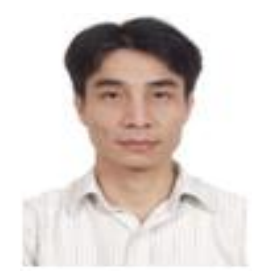

